\title{
RECOMBINANT ERYTHROPOIETIN MITIGATES REPERFUSION INJURY IN NEONATAL RAT CARDIOMYOCYTES BY NOVEL MULTIPLE SIGNALLING PATHWAYS
}

\author{
ASIYA PARVIN ALLAUDEEN ${ }^{1}$, PAVANI KOKA ${ }^{1}$, TARUN PANT ${ }^{1}$, YAMINI CHANDRAMOHAN ${ }^{1}$, \\ SANJANA SIVANESAN ${ }^{2}$, JOHN E. BAKER ${ }^{3}$, ANURADHA DHANASEKARAN ${ }^{*}$
}

\begin{abstract}
${ }^{1}$ Centre for Biotechnology, Anna University, Chennai 600025, Tamil Nadu, India, ${ }^{2}$ SRM University, Porur, Chennai, Tamil Nadu, India, ${ }^{3}$ Department of Surgery, Division of Cardiothoracic Surgery, Medical College of Wisconsin, Milwaukee, Wisconsin, United States of America Email: anu@annauniv.edu
\end{abstract}

Received: 09 Aug 2015 Revised and Accepted: 05 Oct 2016

\begin{abstract}
Objective: Recombinant Human Erythropoietin (rhEPO) is strongly inferred to protect the cardiomyocytes from the reperfusion injury and our aim is to elucidate the cardioprotective effect and the exact mechanism behind the cardioprotection.

Methods: Neonatal rat cardiomyocytes (NCM) exposed to Hypoxia/Reperfusion (H/R) with or without pretreatment using various concentrations of rhEPO. To determine the cell viability-MTT assay, Acridine orange and Ethidium Bromide (Ao/EtBr) staining was performed. To determine the reactive oxygen species (ROS) and mitochondrial membrane potential $(\Delta \psi m)$, Dichlorofluorescein diacetate (DCF-DA) and Rhodamine-123 was used. To determine the signaling pathways Western blot analysis of pAkt, pp38 MAPK, cytochrome-c were performed.

Results: rhEPO was found to reduce the cell death by stabilizing ROS significantly, $\Delta \psi \mathrm{m}$, cytochrome c release, and caspase-3. rhEPO, increases the phosphorylation of p38 MAPK, Akt and BAD compared to H/R. Further myocytes blocked with Wortmannin (WT), and SB203580 showed increased
\end{abstract} caspase-3 activity.

Conclusion: Hence we conclude from this study that rhEPO regulated the factors involved in reperfusion injury through modulation of Akt and p38 MAPK pathways.

Keywords: Neonatal rat cardiomyocytes, Reperfusion injury, Akt, p38 MAPK, rhEPO and BAD

(C) 2016 The Authors. Published by Innovare Academic Sciences Pvt Ltd. This is an open access article under the CC BY license (http://creativecommons.org/licenses/by/4. 0/) DOI: http://dx.doi.org/10.22159/ijpps.2016v8i12.8386

\section{INTRODUCTION}

Heart Failure (HF) is a state in which the heart cannot provide sufficient cardiac output to satisfy the metabolic needs of the body. It is a significant wellbeing issue around the world [1]. HF due to myocardial infarction (MI) or ischemia/reperfusion injury (I/R) results in cardiomyocytes loss. This loss in cardiomyocytes is not only associated with apoptosis but also with necrosis [2-4]. During ischemia/hypoxia because of the lack of oxygen supply, the electron flow gets inhibited, and ATP utilization turns out to be inefficient [5]. Consequently, ATP produced during reperfusion causes partial reduction of oxygen to water producing reactive oxygen species (ROS), which results in damage to the electron transport chain $[6,7]$. Besides ROS, other factors which involved in reperfusion injury are mitochondrial membrane potential $\left(\Delta \psi_{m}\right)$, calcium $\left(\mathrm{Ca}^{2+}\right)$ overload, and cytochrome-c release and caspase cascade activation [8].

Erythropoietin (EPO) is a hypoxia-induced hematopoietic cytokine/ hormone, an erythroid precursor which causes cell proliferation, differentiation and stimulates erythropoiesis. Apart from erythropoiesis, EPO has a cardioprotective effect which increases the number of capillaries and mature vessels in infarcted hearts [9, 10] and up-regulates the expression of angiogenic cytokines such as VEGF and angiopoietin-1 [11-13].

EPO $(3000 \mathrm{U} / \mathrm{Kg})$ has been shown to induce stem cell recruitment and improves cardiac functions when injected by intra-cardiac injection [14]. EPO $(10,000 \mathrm{U} / \mathrm{kg} / \mathrm{d})$ has a protective effect against cardiac remodeling after MI in in vivo mouse models [15]. EPO (1 to $50 \mathrm{U} / \mathrm{ml}$ ) protects $\mathrm{HK}-2$ cells from $\mathrm{H}_{2} \mathrm{O}_{2}$ induced cell death in a dose-dependent manner [16]. A study in rat cardiomyocytes showed protection against Angiotensin II-induced cardiomyocyte hypertrophy when EPO administered at a dose of $20 \mathrm{U} / \mathrm{ml}$ [17]. Moreover, EPO (5000 U/kg) was found to have an anti-apoptotic and anti-necrotic effects in the proximal convoluted tubule when exposed to hypoxia in in vitro in renal I/R injury [18].
EPO (300 IU $/ \mathrm{kg}$ i. v) is able to reduce oxidative stress and caspase-3 activities in $\mathrm{H}_{2} \mathrm{O}_{2}$ induced ischemia in $\mathrm{H} 9 \mathrm{C} 2$ cells and rabbit models [19]. EPO also exerts a neuroprotective effect by attenuating the production of ROS and reducing the basilar artery vasoconstriction on neural, vascular endothelium [20].

We have demonstrated in $\mathrm{H} 9 \mathrm{C} 2$ cells that EPO decreases ROS, $\Delta \psi_{m}$ and intracellular $\mathrm{Ca}^{2+}$ homeostasis via modulation of Akt pathway [21]. Furthermore, it is not known whether EPO has an influence on other factors involved in reperfusion injury, such as caspase activity and cytochrome-c release. We have hypothesized that EPO has an influence on caspase activity, and cytochrome-c release also through modulation of Akt and p38 MAPK pathways in neonatal rat cardiomyocytes (NCM). In the present study, we have therefore investigated the effect of recombinant human erythropoietin (rhEPO) on the apoptosis, necrosis, ROS, $\Delta \psi_{m}$, caspase-3 activity, cytochrome-c release, Akt and p38 MAPK pathways.

\section{MATERIALS AND METHODS}

\section{Isolation of neonatal rat cardiomyocytes}

Our research work on neonatal rat pups was carried out in strict accordance with the recommendations of CPCSEA (committee for the purpose of control and supervision on experiments on animals) guidelines for laboratory animal facility. Our protocol was approved by the institutional animal ethics committee (IAEC) of Anna University (Permit Number: CBT/AU/IAEC 2011-1). All surgery was performed under ethyl ether, and all efforts were made to minimize suffering. All our research work was done in Centre for Biotechnology, Anna University, Taramani Campus, Chennai. Male/Female rat pups (Sprague-Dawley) at the age of 1-3 d were sacrificed by ethyl ether. The hearts were removed and then cut into 1-3 $\mathrm{mm}^{3}$ square pieces and followed the protocol as described in [22]. The cut up tissue was transferred to a $15 \mathrm{ml}$ conical flask containing trypsin solution $(0.08 \%, 0.5 \mathrm{ml}$ per rat) and a small magnetic bead subjected to preconditioning on ice for $20 \mathrm{~min}$. The 
tissue was processed in the conical flask at $37{ }^{\circ} \mathrm{C}$ for $10 \mathrm{~min}$, which was subjected to constant stirring (150-200 rpm). The supernatant was transferred to a $15 \mathrm{ml}$ centrifuge tube, and trypsin activity was inhibited by adding a mixture of trypsin inhibitor and cold culture medium without Bromodeoxyuridine (BrdU), supplemented with $10 \%$ fetal bovine serum (FBS). The cell pellet was formed by centrifuging at $1000 \mathrm{rpm}$ for $5 \mathrm{~min}$ and resuspended in $2 \mathrm{ml}$ warm culture medium. Depending on the amount of undigested tissue, trypsinization and centrifugation steps were repeated 4-5 times. Cells were harvested by centrifugation for $6 \mathrm{~min}$ at $1200 \mathrm{rpm}$ and resuspended in Dulbecco's modified Eagle's medium (DMEM) supplemented with $10 \%$ FBS, plated in tissue culture flask and incubated at $37{ }^{\circ} \mathrm{C}$ at $5 \% \mathrm{CO}_{2}$ for $2 \mathrm{~h}$. This facilitates separation of neonatal cardiomyocytes (NCM) from the non-myocytes (fibroblast). NCM in the supernatant was plated on $0.1 \%$ gelatine coated dishes with coverslips inside and cultured in DMEM containing $0.1 \mathrm{mmol}$ $\mathrm{BrDu}$ (5-Bromo-2-deoxyuridine). BrDu containing DMEM was replaced by DMEM $+10 \%$ FBS after $48 \mathrm{~h}$. Beatings NCM were video graphed on day 3 using Nikon Camera at 20X and 40X.

\section{Characterization of neonatal rat cardiomyocytes}

\section{Hematoxylin and eosin staining method}

NCM was stained with Hematoxylin and Eosin (HandE) to visualize the morphology of NCM clearly. The media were removed from 60 $\mathrm{mm}$ culture dish and NCM were washed using $1 \mathrm{X}$ phosphate buffer saline (PBS). The appropriate volume of $2 \%$ paraformaldehyde was added to the sample and spread properly and kept for 30 min. Paraformaldehyde was removed after $30 \mathrm{~min}$ and hematoxylin was added and incubated for $8 \mathrm{~min}$ and washed with 1X PBS followed by 95\% ethanol wash. Eosin was added to the hematoxylin stained NCM and incubated for 1 min. Then HandE stained NCM were washed with 1X PBS followed by $95 \%$ ethanol. The stained samples were allowed to dry for 5-10 min and images were captured under phase contrast microscope using Nikon microscope.

\section{Immuno-staining method}

NCM were washed with $1 \mathrm{X}$ PBS and incubated for $10 \mathrm{~min}$ at $37^{\circ} \mathrm{C}$ with $2 \%(\mathrm{v} / \mathrm{v})$ paraformaldehyde. Paraformaldehyde was removed carefully and fixed NCM were washed 3 times with 1 XPBS ( 5 min per wash). The sample was permeabilized with Triton X-100 (0.1\%) for 5-10 min at RT (room temperature) then washed in 1XPBS. Samples were incubated at $37^{\circ} \mathrm{C}$ for about 3-4 h with monoclonal Anti- $\alpha$ Sarcomeric Actinin (A7811) (Sigma-Aldrich) at a dilution of 1:200 in $1 \mathrm{X}$ PBS at $37{ }^{\circ} \mathrm{C}$. Samples were washed and incubated with biotinylated anti-mouse secondary antibodies at a dilution of 1:500 dilutions (Santa Cruz Biotechnology) for $45 \mathrm{~min}$ at $37^{\circ} \mathrm{C}$. At the end samples were washed and incubated for $15 \mathrm{~min}$ at $37^{\circ} \mathrm{C}$ with avidinconjugated 1:500 FITC (Fluorescein isothiocyanate) and images were captured using confocal microscopy (Carl Zessis, Zen 2010) with excitation $490 \mathrm{~nm}$ and emission $525 \mathrm{~nm}$ [23].

\section{Treatment of neonatal rat cardiomyocytes}

NCM were cultured to $70 \%-80 \%$ confluency and were allowed to grow in serum-free DMEM $+0.1 \%$ BSA (bovine serum albumin) overnight. Normoxic control group or groups induced to H/R after treatment with or without two applications of rhEPO $(10 \mathrm{U} / \mathrm{ml}, 15$ $\mathrm{U} / \mathrm{ml}$ and $20 \mathrm{U} / \mathrm{ml}$ ) was used in our investigation. The first application of rhEPO was accomplished $24 \mathrm{~h}$ before H/R. A second application of rhEPO immediately before induction of hypoxia and hypoxic conditions were induced by incubating NCM in an airtight chamber $\left(94 \% \mathrm{~N}_{2}, 5 \% \mathrm{CO}_{2}\right.$ and remaining $\left.1 \% \mathrm{O}_{2}\right)$ for $8 \mathrm{~h}$ without serum and glucose in DMEM medium [23]. Reperfusion was induced for $16 \mathrm{~h}$ by replacing glucose containing DMEM+10\% FBS for hypoxic medium. For some of the experiments, cells were treated with $20 \mathrm{U} / \mathrm{ml}$ of EPO alone in normoxic condition. In some experiments, $10 \mu \mathrm{M}$ SB203580, p38 MAPK inhibitor and $1 \mu \mathrm{M}$ Wortmannin (WT), PI3K/AKT inhibitor were added 30 min prior to each application of rhEPO. Control cells were maintained in DMEM $+10 \%$ FBS throughout the duration of the experiments.

\section{MTT assay}

NCM were cultured in $0.1 \%$ gelatine coated 96 well plates for about $70-80 \%$ confluency. NCM were pretreated with different concentration (10 U/ml, $15 \mathrm{U} / \mathrm{ml}$ and $20 \mathrm{U} / \mathrm{ml}$ of rhEPO) and incubated for $24 \mathrm{~h}$ and followed the treatment as described in the treatment of NCM. The MTT [3-(4, 5-dimethylthiazole-2-yl)-2, 5diphenyltetrazolium bromide] were added to each well and incubated at $37^{\circ} \mathrm{C}$ in a $\mathrm{CO}_{2}$ incubator for $4 \mathrm{~h}$ [24]. Soluble yellow color MTT is reduced by mitochondrial succinyl dehydrogenase into insoluble purple formazan. The insoluble formazan product was dissolved in $50 \mu \mathrm{l}$ DMSO and incubated for $10 \mathrm{~min}$ and read absorbance at $540 \mathrm{~nm}$.

\section{Detection of apoptosis and necrosis}

Followed by the pretreatment NCM were washed with 1XPBS and collected by centrifugation. NCM were resuspended in $100 \mu \mathrm{l}$ of 1XPBS, $10 \mu \mathrm{l}$ of the cell suspension were put on a glass slide and then mixed with $1 \mu \mathrm{l}$ of $1 \mathrm{mg} / \mathrm{ml}$ Acridine orange (Ao) and $1 \mu \mathrm{l}$ of 10 $\mu \mathrm{g} / \mathrm{ml}$ Ethidium Bromide (EtBr). The coverslip was placed over the glass slide and immediately viewed under a confocal laser scanning microscope at a wavelength of excitation $502 \mathrm{~nm}$ and emission 526 $\mathrm{nm}$ for Ao and for EtBr excitation $510 \mathrm{~nm}$ and emission $595 \mathrm{~nm}$ [21].

\section{Detection of mitochondrial membrane potential and ROS}

After pretreatment NCM were cultured on $0.1 \%$ gelatine coated coverslips were washed with 1 XPBS and incubated with Rhodamine- 123 $(5 \mu \mathrm{g} / \mathrm{ml})$ and DCFH-DA $(100 \mu \mathrm{M})$ reagent for $30 \mathrm{~min}$ in the dark at 37 ${ }^{\circ} \mathrm{C}$. Samples were washed thrice with 1XPBS and then the fluorescence was measured using a confocal laser scanning microscope using $514 \mathrm{~nm}$ and $570 \mathrm{~nm}$ for Rhodamine-123 and DCF-DA respectively. Before that confocal laser scanning microscope adjusts to the laser power $(2 \%)$, detector gain, and resolution $(512 \times 512)$ and focused it on $40 \mathrm{X}$ oil immersion. The same parameter is used for all sets of experiments [21]. Quantification of the fluorescence was done by using image J software and the corrected cell fluorescence (CTCF) was obtained using the formula, CTCF = Integrated Density-(Area of selected cell X Mean fluorescence of background readings).

\section{Western blot analysis}

NCM were cultured on gelatin ( $0.1 \%)$ coated autoclaved glass coverslips in $60 \mathrm{~mm}$ dishes and was treated under $8 \mathrm{~h}$ hypoxia and $30 \mathrm{~min}$ of reperfusion with or without EPO. Cells were kept on ice and washed thrice with cold 1 XPBS. Proteins were solubilized and extracted with 50 $\mu$ l RIPA buffer (50 mmol Tris, pH 8.0, $150 \mathrm{mmol} \mathrm{NaCl}, 0.5 \%$ SDS, $1 \%$ Nonidet P40, $0.5 \%$ sodium deoxycholate, 1 mmol EDTA, 1 X protease and phosphatase inhibitor cocktail (Cell Signaling technologies.). The lysate was utilized to estimate protein content with the Bradford Assay Reagent. Equal amounts of protein $(20-50 \mu \mathrm{g})$ from each sample were electrophoresed on a $12 \%$ SDS-polyacrylamide gel with running buffer and transferred to a nitrocellulose membrane as described in the Cell Signaling technology protocol. The transferred membranes were checked with ponceau and incubated with primary antibodies such as Akt, p-Akt, p38 MAPK, pp38 MAPK, BAD, p-BAD (1:1000 dilutions, Cell Signaling Technologies.,) for overnight incubation in $4{ }^{\circ} \mathrm{C}$. They were again washed 3 times with 1xTBST (Tris-Buffered Saline and Tween 20) before incubating with matching secondary antibody (1:10 000) for 45 min. The protein bands were developed with alkaline phosphatase substrate.

\section{Detection of cytochrome c releases into cytosol}

The release of mitochondrial cytochrome-c into the cytosol was measured according to the protocol described previously [25]. Cultured NCM were rinsed with 1XPBS and homogenized in $0.25 \mathrm{M}$ sucrose, $20 \mathrm{mmol}$ Tris- $\mathrm{HCl}$, and $5 \mathrm{mmol}$ EDTA, $\mathrm{pH}$ 7. The homogenates were centrifuged at $800 \mathrm{~g}$, discarded the pellet and the supernatant was centrifuged at $8000 \mathrm{~g}$ for $10 \mathrm{~min}$. The pellet contains the mitochondrial fraction and the supernatant contains a soluble cytosol-enriched fraction. The supernatant was collected to measure cytochrome c released into the cytosol and the pellet was resuspended for western blot analysis using anti-rabbit cytochrome$\mathrm{c}$ as described previously in Western blot analysis.

\section{Detection of caspase-3 enzymatic activity}

NCM were cultured in $0.1 \%$ gelatine coated $60 \mathrm{~mm}$ cell culture dishes for about $70-80 \%$ confluency. After induction of $H / R$ with or 
without pretreatment with rhEPO, myocytes were washed with 1XPBS and lysed with cell lysis buffer. The enzymatic activity of the caspase- 3 is measured by following the protocol given in the caspase- 3 colorimetric assay kit, RandD systems. After that, the cell lysate was incubated for $10 \mathrm{~min}$ on ice and centrifuged at $10000 \mathrm{x} \mathrm{g}$ for $1 \mathrm{~min}$. The supernatant was transferred to a new tube and kept on ice. Then $50 \mu \mathrm{l}$ of the supernatant was added to $50 \mu \mathrm{l}$ of $2 \mathrm{X}$ Reaction buffer containing DTT in 96 well plates. At the end, reaction mixtures were incubated with $5 \mu \mathrm{l}$ of DEVD-pNA (caspase-3 colorimetric substrate) for 1-2 h. The caspase activities were quantified by using a spectrofluorometer using a wavelength of 405 $\mathrm{nm}[26]$.

\section{Statistics}

Statistical data were analyzed using ANOVA followed by TUKEYs tests in Graph Pad Prism. For all the experiments, data are presented as means \pm SEM from three to five samples. Values of $\mathrm{P}<0.05$ were considered as statistically significant.

\section{RESULTS}

\section{Characterization of NCM}

The NCM were isolated from 2 d-old neonatal rats. The morphology of the NCM was examined under an inverted microscope (Nikon) (fig. 1A). The cells were stained with nuclear stain hematoxylin and cytoplasmic stain Eosin and viewed under the microscope (fig. 1B). The beating of the cells was also observed during the third day after isolation. The beating was recorded to be 61 beats per min. The isolated cells were also confirmed to be cardiomyocytes by indirect immunofluorescence assay using anti- $\alpha$-sarcomeric actin antibody which is a specific cardiac marker and showed green color fluorescence (fig. 1C).
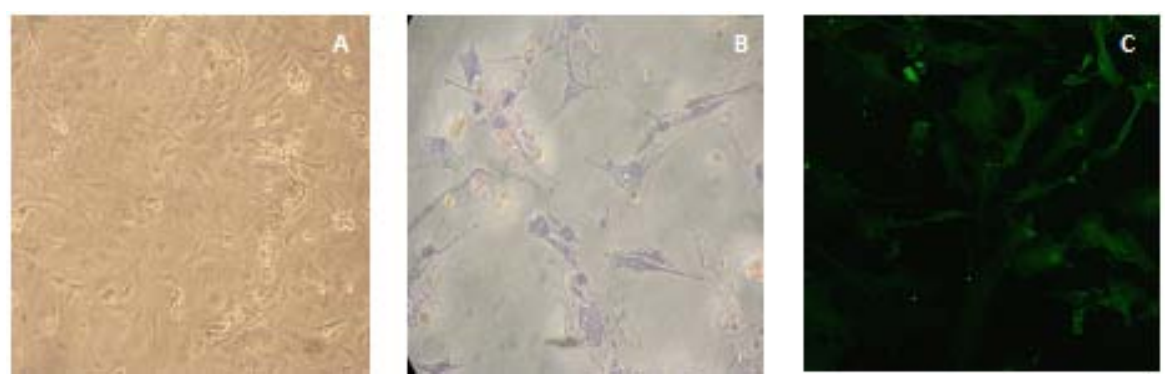

Fig. 1: Characterization of neonatal cardiomyocytes

\section{rhEPO cell viability studies}

NCM demonstrated increased survival rates when maintained under normoxia or pretreated with $20 \mathrm{U} / \mathrm{ml}$ as compared to the NCM which were subjected to $\mathrm{H} / \mathrm{R}$ alone. Cell viability was increased from $45 \%$ in H/R injured NCM to $83.5 \%$ of $20 \mathrm{U} / \mathrm{ml}$ rhEPO pretreated NCM. Data are presented as means \pm SEM of the ratios from five independent experiments.* denotes $\mathrm{p}<0.05$ for analyses compared to $\mathrm{H} / \mathrm{R}$ (fig. 2).

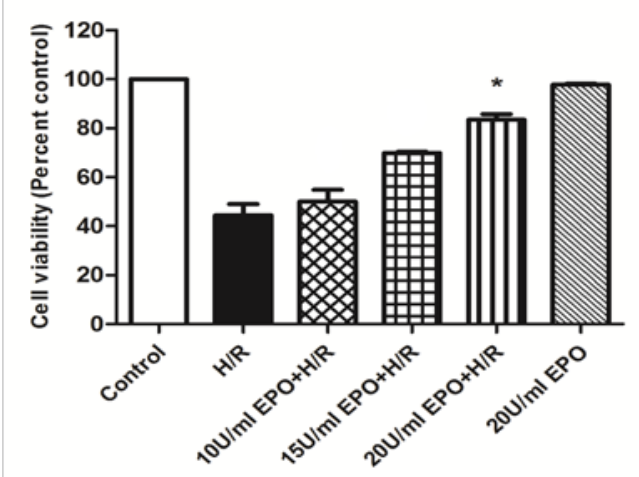

Fig. 2: Pre-treatment of rhEPO Increases Cell Viability in H/RInduced NCM

\section{rhEPO inhibits apoptosis and necrosis in H/R-induced NCM}

To distinguish viable cells from apoptotic and necrotic cells, Ao and EtBr double staining method was used. Control and rhEPO treated myocytes stained uniform green color because they can maintain membrane integrity (fig. $3 \mathrm{~A}$ and 3C). Whereas H/R-induced myocytes lost membrane integrity so $\mathrm{EtBr}$ could enter and intercalated with DNA. Late apoptotic myocytes showed bright orange nuclei and necrotic myocytes showed red nuclei as showed in the fig. $3 B$. Data are presented as means \pm SEM of the ratios from three independent experiments.* denotes $\mathrm{p}<0.05$ for analyses compared to H/R.

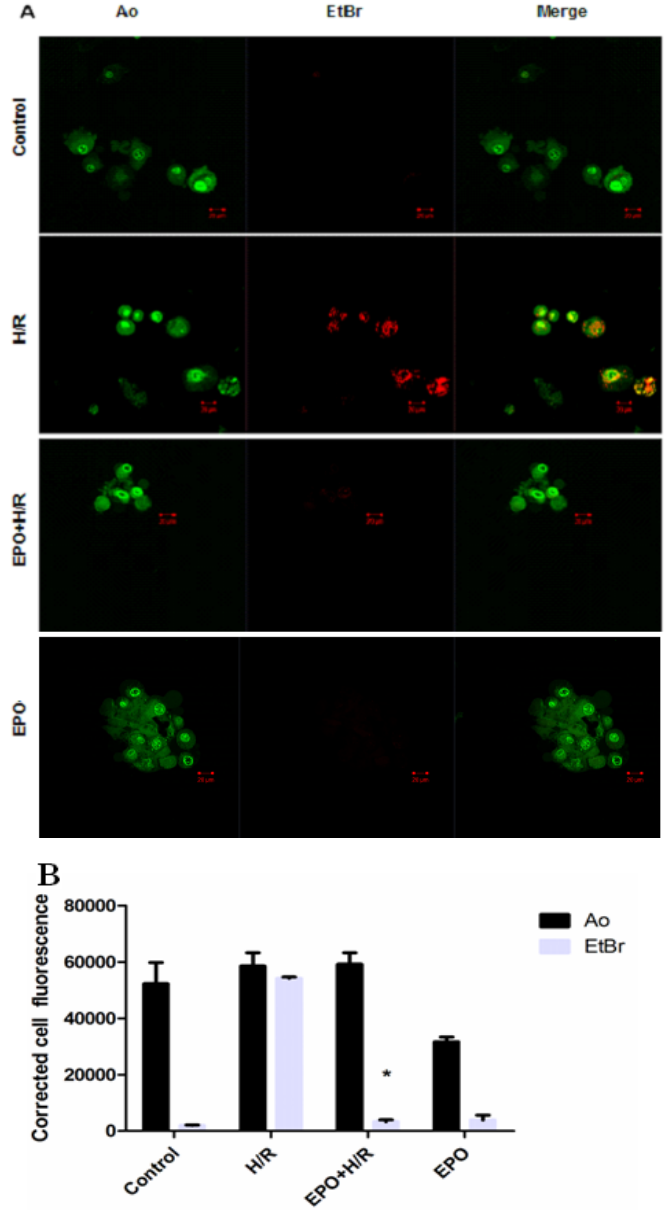

Fig. 3: Pre-treatment of rhEPO in H/R-induced NCM had uniformly green nuclei with intact plasma and nuclear membranes 


\section{rhEPO stabilizes $\Delta \Psi \mathrm{m}$ and ROS in $\mathrm{H} / \mathrm{R}$-induced NCM}

In control and rhEPO treated myocytes, fluorescence emitted by Rhodamine-123 appeared only in the perinuclear region where the mitochondria is located as showed in the fig. 4A and 4C. DCFH-DA crosses the cell membranes and the mitochondrial membrane by deacetylation and oxidation. This is facilitated by esterases and ROS in the cytoplasm and mitochondria. In H/R-induced NCM, Rhodamine-123 fluorescence colocalized with DCF fluorescence not only the perinuclear region but also in the cytoplasmic region and there was an increase in DCF fluorescence in H/R when compared to rhEPO pretreated NCM (fig. 4B). Data are presented as means \pm SEM of the ratios from three independent experiments.* denotes $\mathrm{p}<0.05$ for analyses compared to $\mathrm{H} / \mathrm{R}$. Accordingly rhEPO stabilizes $\Delta \psi_{m}$ and ROS in H/R-induced NCM.

A
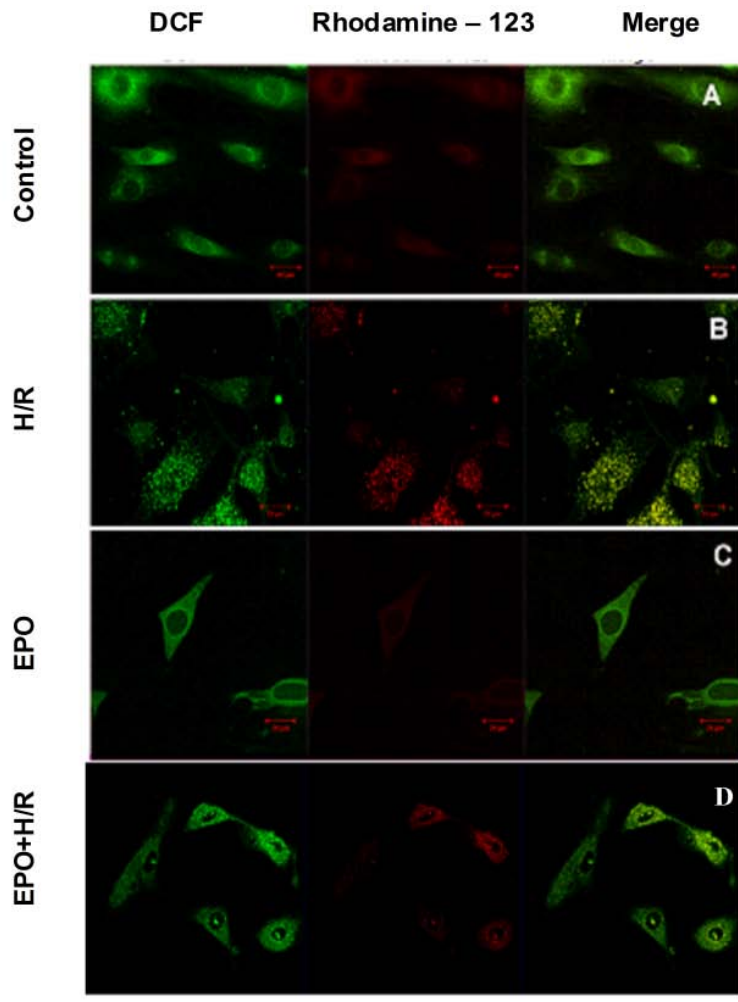

B

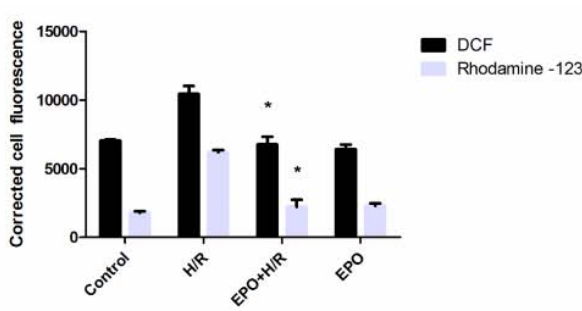

Fig. 4: Pre-treatment of rhEPO stabilizes ROS and $\Delta \psi_{m}$ in H/RInduced NCM

\section{rhEPO induces phosphorylation of Akt}

Fig. 5A depicts the phosphorylation of Akt in H/R-induced myocytes. NCM pretreated with rhEPO post-H/R showed a significant increase in phosphorylation of AKT in lane 3 of pAkt as compared to cells exposed to H/R. Expression of AKT in the corresponding row is not altered. This increase in phosphorylation of AKT was blocked by WT. Data are presented as means \pm SEM of the ratios from three independent experiments. ${ }^{*}$ denotes $\mathrm{p}<0.05$ for analyses compared to $\mathrm{H} / \mathrm{R}$.

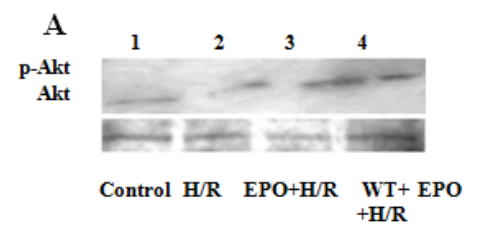

B

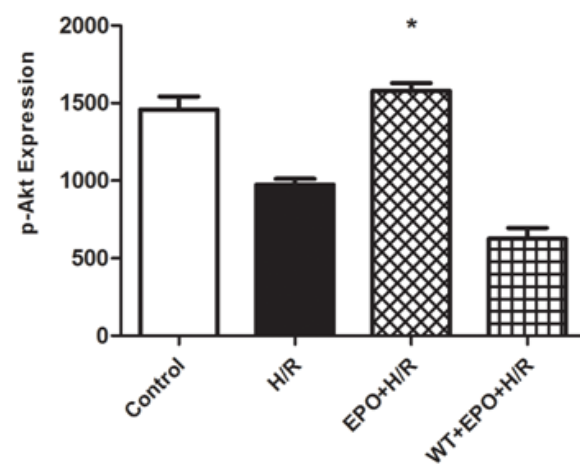

Fig. 5: Western blot analysis demonstrating the effect of rhEPO on Akt

\section{rhEPO increases the phosphorylation of p38 MAPK}

Fig. 6A depicts the phosphorylation of p38 MAPK in H/R-induced myocytes. NCM pretreated with rhEPO showed an increase in p38 MAPK phosphorylation in lane 3 when compared to control and H/R. The phosphorylation of p38 MAPK was blocked by SB203580. Expression of p38 MAPK (loading control) in the corresponding row is not altered. Data are presented as means \pm SEM of the ratios from three independent experiments. ${ }^{*}$ denotes $\mathrm{p}<0.05$ for analyses compared to H/R.

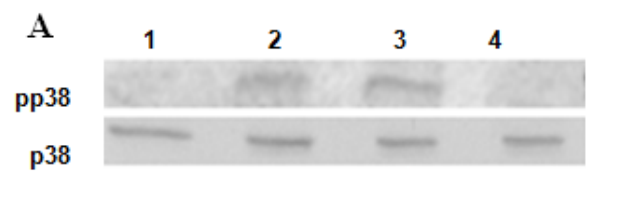

Control H/R $\quad \mathrm{EPO}+\mathrm{H} / \mathrm{R} \quad \mathrm{SB}+\mathrm{EPO}+\mathrm{H} / \mathrm{R}$

$\mathbf{B}$

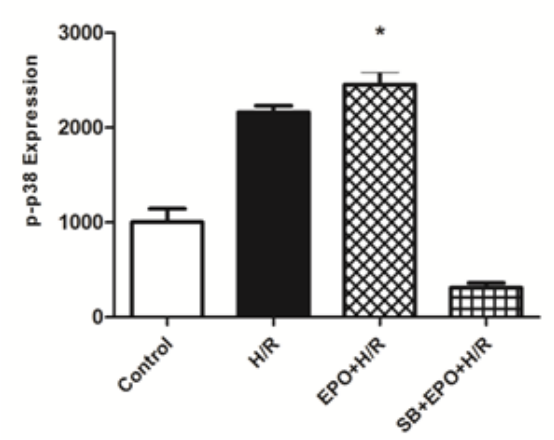

Fig. 6: Western blot analysis demonstrating the effect of rhEPO on P38 MAPK

\section{rhEPO increases the phosphorylation of BAD}

Fig. 7A depicts the phosphorylation of BAD in H/R-induced myocytes. The myocytes pretreated with rhEPO showed a significant increase in phosphorylation of BAD in lane 3 as compared to myocytes exposed to H/R without rhEPO pretreatment. Expression of BAD (loading control) in the corresponding row is not altered. Data are presented as means \pm SEM of the ratios from three independent experiments.* denotes $\mathrm{p}<0.05$ for analyses compared to $\mathrm{H} / \mathrm{R}$. 


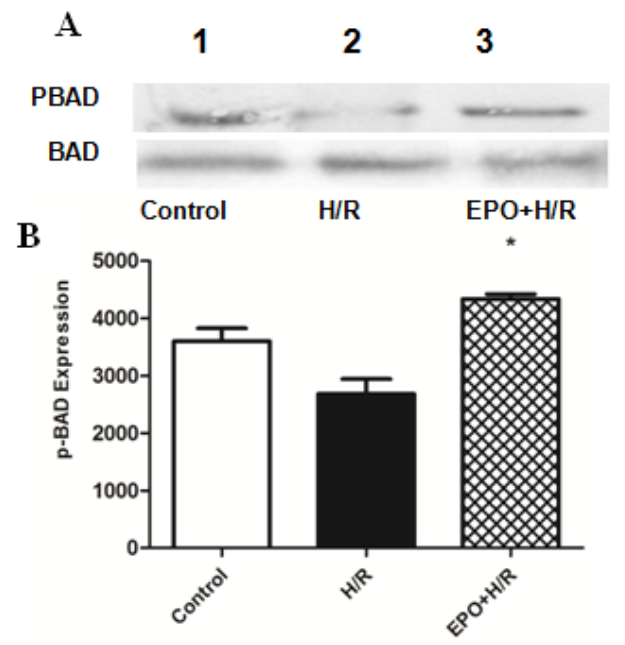

Fig. 7: Western blot analysis demonstrating the effect of rhEPO on BAD

\section{rhEPO decreases cytosolic release of cytochrome-c}

Fig. 8A depicts the cytosolic and mitochondrial release of cytochrome-c in H/R-induced myocytes. rhEPO pretreated NCM showed decrease cytosolic release of cytochrome-c (cytosolic cytochrome-c, lane 3) and increased mitochondrial accumulation of cytochrome-c (mitochondrial cytochrome-c, lane 3) but NCM without rhEPO pretreatment showed increased cytosolic release of cytochrome-c (cytosolic cytochrome-c, panel 2) and decreased mitochondrial accumulation of cytochrome-c (mitochondrial cytochrome-c, lane 2). Data are presented as means \pm SEM of the ratios from three independent experiments.* denotes $\mathrm{p}<0.05$ for analyses compared to $H / R$.

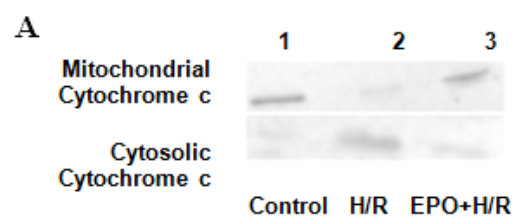

B

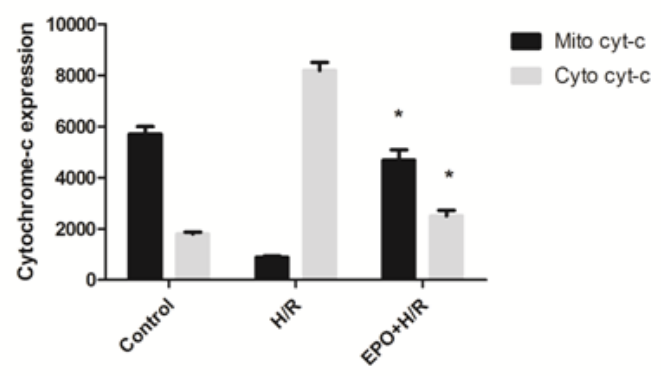

Fig. 8: Western blot analysis demonstrating the effect of rhEPO on cytochrome-c

\section{rhEPO inhibits caspase-3 activity in NCM}

NCM were induced with $\mathrm{H} / \mathrm{R}$ with or without rhEPO treatment and caspase- 3 activity was measured. The caspase- 3 activities were markedly elevated after H/R. Pretreatment with rhEPO prevented the caspase- 3 proteolytic activations induced by H/R. Further myocytes blocked with WT and SB203580 showed increase caspase3 activity as showed in fig. 9. Data are presented as means \pm SEM of the ratios from three independent experiments.* denotes $p<0.05$ for analyses compared to $\mathrm{H} / \mathrm{R}$.

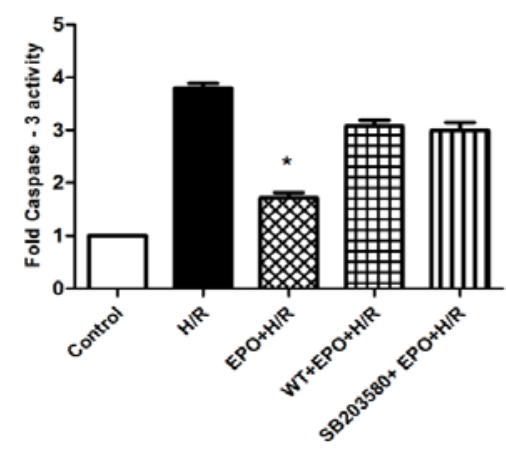

Fig. 9: Pre-treatment of rhEPO decreases caspase-3 activity

\section{DISCUSSION}

We believe our study is the first to demonstrate that the rhEPO regulates the factors involved in reperfusion injury, such as ROS, $\Delta \psi \mathrm{m}$, cytochrome-c release and caspase- 3 activity and protects NCM from cell death. We also found that the regulation was mediated through the modulation of Akt and p38 MAPK.

Reperfusion of coronary artery flow is imperative to resuscitate the hypoxic/ischemic myocardium. Timely reperfusion encourages cardiomyocyte rescue and decreases cardiac morbidity and mortality [27]. Reperfusion rescues myocytes inside ischemic areas of necrosis, however, it causes lethal damage to myocytes with serious ischemiainduced metabolic disturbances [28]. It is known that ischemia and reperfusion-induced ventricular arrhythmias and post-ischemic myocardial dysfunction (myocardial stunning), microvascular and endothelial injury [29-31] leads to reperfusion injury. Further reperfusion injury involves myocyte damage through apoptosis and irreversible cell damage or necrosis [32,33]. Administration of EPO reduces necrosis in an ischemic myocutaneous tissue in a dosedependent manner [34]. EPO treatment before or at the start of ischemia has been demonstrated to decrease apoptosis and ventricular dysfunction after I/R injury [35-37]. However, a decrease in necrosis was thus induced to protect from reperfusion injury, and rhEPO was efficiently used in this study by showing a reduction in both apoptosis and necrosis caused by reperfusion injury. In support to our present study, a clinical trial showed an effective protection of EPO against tissue injury caused by both apoptosis and necrosis following 6 mo of human kidney transplantations [38].

MPTP is the mega channel in the inner mitochondrial membrane it allows molecules of $<1.5 \mathrm{kDa}$ when it opens [39]. MPTP opening is of two sorts: one is transient or intermediate, and another is longlasting opening, this variation depends on the balance between the cellular inducers and inhibitors [40]. I/R causes longlasting/irreversible opening of MPTP and the major consequence of long-lasting MPTP opening is destabilization of $\Delta \psi_{\mathrm{m}}$, depletion of ATP and NAD+, increased ROS generation, swelling of mitochondrial matrix, release of accumulated $\mathrm{Ca}^{2+}$, outer mitochondrial membrane rupture and cytochrome-c release, which further activates caspase cascade and blocks the electron flow via the electron transport chain [39,41-42]. Inhibition of these factors was in turn inferred to attenuate cell death, and rhEPO was effectively used in our present study in demonstrating significant decreases in the levels of ROS production, cytochrome-c release, caspase- 3 activation and stabilization of $\Delta \psi_{m}$, in NCM upon exposure to H/R. EPO has been found to exert neuroprotective effects by attenuating the production of ROS and reducing the basilar artery vasoconstriction on neural vascular endothelium [20].

Activation of Akt signaling pathway results in activation of antiapoptotic factors such as eNOS, p70S6K and also the inhibition of pro-apoptotic factors such as caspase, BIM, BAD and BAX [43]. EPO has been reported to protect acute neuronal injury through the phosphorylation of Akt, Bad, maintaining $\Delta \psi_{m}$ and nuclear neuronal DNA integrity and simultaneously inhibiting caspase-1, 3 and 8 activities [44]. In our study rhEPO pretreatment showed a protective effect by a decrease in caspase- 3 activity, and the mechanism of 
inhibition is inferred to be through the modulation of pro-survival signaling pathway Akt. Evidence for this mechanism was confirmed by blocking the Akt pathway using WT and an increased in caspase3 activity was observed and thus confirming that the effect is primarily due to phosphorylation of Akt.

Previous studies have shown HPC (Hypoxic Preconditioning) induced neuroprotection in cerebral ischemic injury is through the phosphorylation of p38 MAPK pathway [45]. Evidence suggested that EPO and HPC target the same signaling pathway [46] and this is confirmed by blocking the EPOR during hypoxia, which results in reduced HPC-induced effects such as activation of caspase-3, increased expression of BCL-2 and cell survival [47]. Thus, the use of EPO as a "Chemical preconditioning" acts as an alternative to HPC for promoting cell survival in embryonic stem cells transplanted into the ischemic rat brain $[46,48]$. Our present data showed an increase in phosphorylation of p38 MAPK in rhEPO pretreated NCM confirmed the previous studies, that rhEPO protection against H/R in NCM through the phosphorylation of p38 MAPK. The phosphorylation of p38 MAPK was blocked with SB203580 (p38 MAPK inhibitor) and decreased caspase- 3 activity.

One of the downstream targets of Akt is BCL-2-associated death promoter (BAD). BAD belongs to the BCL-2 family and it includes both anti-apoptotic proteins and pro-apoptotic protein. When Akt phosphorylates BAD, it forms the BAD-(14-3-3) protein homodimer and allows BCL-2 which is free to inhibit apoptosis triggered by BAX. $\mathrm{BAD}$ phosphorylation is anti-apoptotic, and dephosphorylation is pro-apoptotic [49].

\section{CONCLUSION}

In conclusion, we demonstrate that rhEPO prevents apoptosis and necrosis in NCM subjected to H/R injury through increased phosphorylation of Akt, BAD and p38 MAPK. Our study shows for the first time that rhEPO pretreatment maintains $\Delta \psi_{m}$ and decreases the production of ROS, caspase- 3 activity and release of cytochromec from mitochondria into the cytosol in H/R-induced NCM. Thus, our observations support mechanistic evidence for the protective effect of the rhEPO in H/R-induced NCM cell death.

\section{ACKNOWLEDGMENT}

Financial support was provided by the Department of Science and Technology.

\section{CONFLICTS OF INTERESTS}

Declared none

\section{REFERENCES}

1. Gao E, Koch WJ. Is erythropoietin behind maladaptive anemic heart failure? Am J Physiol-Heart C 2009;296:H559-H60.

2. Junk AK, Mammis A, Savitz SI, Singh M, Roth S, et al. Erythropoietin administration protects retinal neurons from acute ischemiareperfusion injury. Proc Natl Acad Sci 2002;99:10659-64.

3. Malhotra R, Lin Z, Vincenz C, Brosius Iii FC. Hypoxia induces apoptosis via two independent pathways in Jurkat cells: differential regulation by glucose. Am J Physiol Cell Physiol 2001;281:C1596-C603.

4. Lemasters JJ, Nieminen AL, Qian T, Trost LC. The mitochondrial permeability transition in cell death: a common mechanism in necrosis, apoptosis and autophagy. BBA-Bioenergetics 1998;1366:177-96.

5. Grover GJ, Atwal KS, Sleph PG, Wang FL. Excessive ATP hydrolysis in ischemic myocardium by mitochondrial F1F0ATPase: effect of selective pharmacological inhibition of mitochondrial ATPase hydrolase activity. Am J Physiol Heart C 2004;287:H1747-H55.

6. Giorgio M, Migliaccio E, Orsini F, Paolucci D. Electron transfer between cytochrome $\mathrm{c}$ and $\mathrm{p} 6$ generates reactive oxygen species that trigger mitochondrial apoptosis. Cell 2005;122:221-33.

7. Zweier JL, Talukder MAH. The role of oxidants and free radicals in reperfusion injury. Cardiovasc Res 2006;70:181-90.

8. Murphy E, Steenbergen C. Mechanisms underlying acute protection from cardiac ischemia-reperfusion injury. Physiol Rev 2008;88:581-609.
9. Boucher M, Pesant S, Lei YH, Nanton N. Simultaneous administration of insulin-like growth factor-1 and darbepoetin alfa protects the rat myocardium against myocardial infarction and enhances angiogenesis. Clin Transl Sci 2008;1:13-20.

10. van der Meer P, Lipsic E, Henning RH, Boddeus K. Erythropoietin induces neovascularization and improves cardiac function in rats with heart failure after myocardial infarction. J Am Coll Cardiol 2005;46:125-33.

11. Maxwell PH, Osmond MK, Pugh CW, Heryet A. Identification of the renal erythropoietin-producing cells using transgenic mice. Kidney Int 1993;44:1149-62.

12. Maxwell PH, Pugh CW, Ratcliffe PJ. The inducible operation of the erythropoietin 3' enhancer in multiple cell lines: evidence for a widespread oxygen-sensing mechanism. Proc Natl Acad Sci 1993;90:2423-7.

13. Wenger RH. Mammalian oxygen sensing, signaling and gene regulation. J Exp Biol 2000;203:1253-63.

14. Klopsch C, Furlani D, Gäbel R, Li W, et al. Intracardiac injection of erythropoietin induces stem cell recruitment and improves cardiac functions in a rat myocardial infarction model. J Cell Mol Med 2009;13:664-79.

15. Ueda K, Takano H, Niitsuma Y, Hasegawa H. Sonic hedgehog is a critical mediator of erythropoietin-induced cardiac protection in mice. J Clin Invest 2010;120:2016-29.

16. Nagasaki Y, Yoshitomi T, Hirayama A, Schock-Kusch D. Acute kidney injury-Experimental models: NDT Plus; 2011. p. 4.

17. Wen Y, Zhang XJ, Ma YX, Xu XJ. Erythropoietin attenuates hypertrophy of neonatal rat cardiac myocytes induced by angiotensin-II in vitro. Scand J Clin Lab Invest 2009;69:518-25.

18. Sharples EJ, Patel N, Brown P, Stewart K. Erythropoietin protects the kidney against the injury and dysfunction caused by ischemia-reperfusion. J Am Soc Nephrol 2004;15:2115-24.

19. Abdelrahman M, Sharples EJ, McDonald MC, Collin M. Erythropoietin attenuates the tissue injury associated with hemorrhagic shock and myocardial ischemia. Shock 2004;22:63-9.

20. Schmeding M, Hunold G, Ariyakhagorn V, Rademacher S. Erythropoietin reduces ischemia-reperfusion injury after liver transplantation in rats. Transplant Int 2009;22:738-46.

21. Parvin A, Raj Pranap A, Shalini U, Devendran A, Baker JE, Dhanasekaran A. Erythropoietin protects cardiomyocytes from cell death during hypoxia/reperfusion injury through activation of survival signaling pathways. PloS One 2014;9:e107453.

22. Fu J, Gao J, Pi R, Liu P. An optimized protocol for the culture of cardiomyocyte from a neonatal rat. Cytotechnology 2005;49:109-16.

23. Dhanasekaran A, Gruenloh SK, Buonaccorsi JN, Zhang R. Multiple antiapoptotic targets of the PI3K/Akt survival pathway are activated by epoxyeicosatrienoic acids to protect cardiomyocytes from hypoxia/anoxia. Am J Physiol-Heart C 2008;294:H724-H35.

24. Lingadurai S, Roy S, Joseph RV, Nath LK. Antileukemic activity of the leaf extract of Bischofia javanica blume on human leukemic cell lines. Indian J Pharmacol 2011;43:143-9.

25. Jin ZQ, Zhou HZ, Cecchini G, Gray MO, Karliner JS. MnSOD in mouse heart: acute responses to ischemic preconditioning and ischemiareperfusion injury. Am J Physiol-Heart C 2005;288:H2986-H94.

26. Dhanasekaran A, Bodiga S, Gruenloh S, Gao Y. 20-HETE increases survival and decreases apoptosis in pulmonary arteries and pulmonary artery endothelial cells. Am J PhysiolHeart C 2009;296:H777-H86

27. Verma S, Fedak PWM, Weisel RD, Butany J, Rao V, Maitland A, et al. Fundamentals of reperfusion injury for the clinical cardiologist. Circulation 2002;105:2332-6.

28. Miura T, Tanno M, Sato T. Mitochondrial kinase signaling pathways in myocardial protection from ischaemia/reperfusion-induced necrosis. Cardiovasc Res 2010;88:7-15.

29. Vegh A, Komori S, Szekeres L, Parratt JR. Antiarrhythmic effects of preconditioning in anesthetized dogs and rats. Cardiovasc Res 1992;26:487-95.

30. Sun HY, Wang NP, Halkos M, Kerendi F. Postconditioning attenuates cardiomyocyte apoptosis via inhibition of JNK and p38 mitogen-activated protein kinase signaling pathways. Apoptosis 2006;11:1583-93. 
31. Takano H, Tang XL, Kodani E, Bolli R. Late preconditioning enhances recovery of myocardial function after infarction in conscious rabbits. Am J Physiol-Heart C 2000;279:H2372-H81.

32. Yellon DM, Baxter GF. Protecting the ischaemic and reperfused myocardium in acute myocardial infarction: distant dream or near reality? Heart 2000;83:381-7.

33. Ambrosio G, Tritto I. Reperfusion injury: experimental evidence and clinical implications. Am Heart J 1999;138:S69-S75.

34. Harder Y, Amon M, Schramm R, Contaldo C. Erythropoietin reduces necrosis in critically ischemic myocutaneous tissue by protecting nutritive perfusion in a dose-dependent manner. Surgery 2009;145:372-83.

35. Fiordaliso F, Chimenti S, Staszewsky L, Bai A. A nonerythropoietic derivative of erythropoietin protects the myocardium from ischemia-reperfusion injury. Proc Natl Acad Sci 2005;102:2046-51.

36. Calvillo L, Latini R, Kajstura J, Leri A. Recombinant human erythropoietin protects the myocardium from ischemiareperfusion injury and promotes beneficial remodeling. Proc Natl Acad Sci 2003;100:4802-6.

37. Cai Z, Semenza GL. Phosphatidylinositol-3-kinase signaling is required for erythropoietin-mediated acute protection against myocardial ischemia/reperfusion injury. Circulation 2004;109:2050-3.

38. Fliser D. Erythropoietin (EPO) and Ischemia-reperfusion After Kidney Transplantation. 2010 ed. Germany: Clinical Trials. gov Archive Site; 2010.

39. Zorov DB, Juhaszova M, Yaniv Y, Nuss HB, Wang S, Sollott SJ. Regulation and pharmacology of the mitochondrial permeability transition pore. Cardiovasc Res 2009;83:213-25.

40. Petronilli V, Penzo D, Scorrano L, Bernardi P. The mitochondrial permeability transition, the release of cytochrome $\mathrm{c}$ and cell death correlation with the duration of pore openings in situ. J Biol Chem 2001;276:12030-4.

41. Bernardi P, Krauskopf A, Basso E, Petronilli V. The mitochondrial permeability transition from in vitro artifact to disease target. FASEB J 2006;273:2077-99.
42. Di Lisa F, Menabò R, Canton M, Barile M. Opening of the mitochondrial permeability transition pore causes depletion of mitochondrial and cytosolic NAD+and is a causative event in the death of myocytes in postischemic reperfusion of the heart. J Biol Chem 2001;276:2571-5.

43. Rafiee P, Shi Y, Su J, Pritchard KA. Erythropoietin protects the infant heart against ischemia-reperfusion injury by triggering multiple signaling pathways. Basic Res Cardiol 2005;100:187-97.

44. Chong ZZ, Kang JQ, Maiese K. Erythropoietin fosters both intrinsic and extrinsic neuronal protection through modulation of microglia, Akt1, Bad, and caspase-mediated pathways. Br J Pharmacol 2003;138:1107-18.

45. Zhao L, Liu X, Liang J, Han S. Phosphorylation of p38 MAPK mediates hypoxic preconditioning-induced neuroprotection against cerebral ischemic injury via mitochondrial translocation of Bcl-xL in mice. Brain Res 2013;1503:78-88.

46. Theus $\mathrm{MH}$, Wei L, Cui L, Francis K. In vitro hypoxic preconditioning of embryonic stem cells as a strategy of promoting cell survival and functional benefits after transplantation into the ischemic rat brain. Exp Neurol 2008;210:656-70.

47. Baker JE, Curry BD, Olinger GN, Gross GJ. Increased tolerance of the chronically hypoxic immature heart to ischemia: contribution of the KATP channel. Circulation 1997;95:1278-85.

48. Prass K, Scharff A, Ruscher K, Löwl D. Hypoxia-induced stroke tolerance in the mouse is mediated by erythropoietin. Stroke 2003;34:1981-6.

49. Foster TC, Sharrow KM, Masse JR, Norris CM. Calcineurin links Ca2+dysregulation with brain aging. J Neurosci 2001;21:4066-73.

\section{How to cite this article}

- Asiya Parvin Allaudeen, Pavani Koka, Tarun Pant, Yamini Chandramohan, Sanjana Sivanesan, John E Baker, Anuradha Dhanasekaran. Recombinant erythropoietin mitigates reperfusion injury in neonatal rat cardiomyocytes by novel multiple signaling pathways. Int J Pharm Pharm Sci 2016;8(12):34-40. 